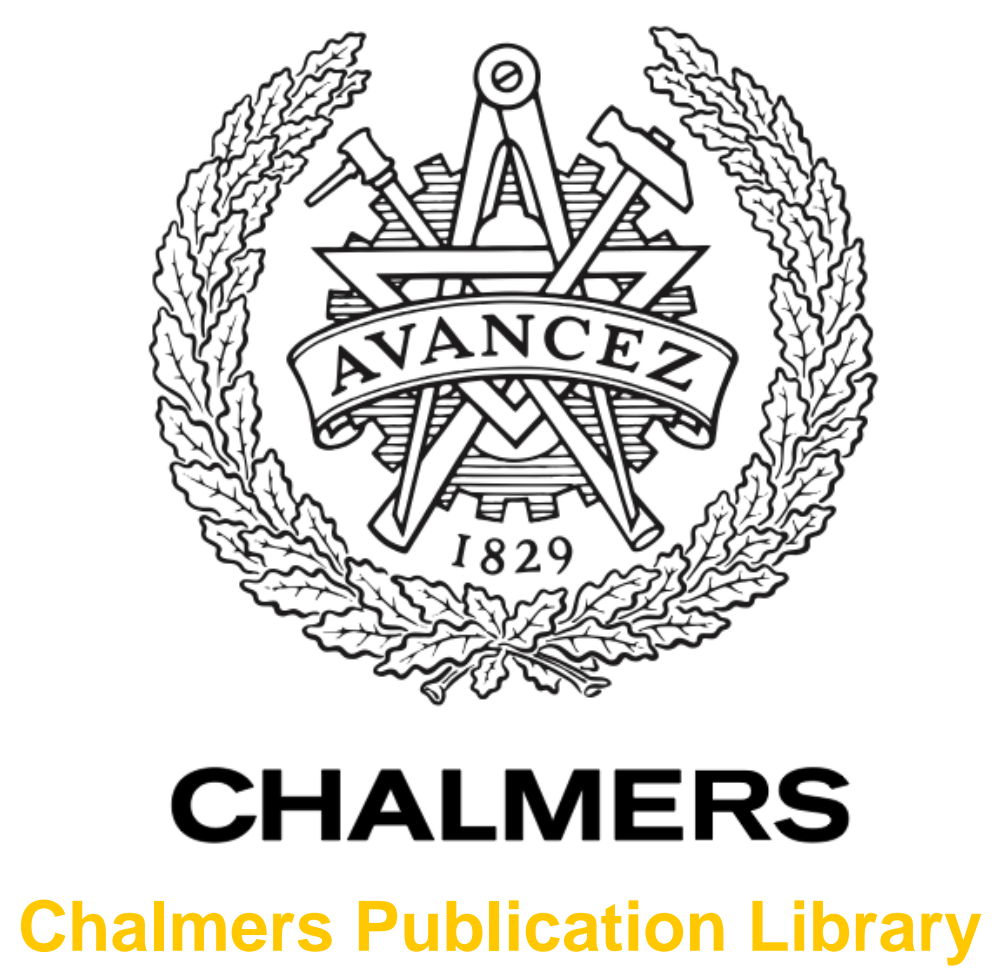

\title{
Concept of a mixer based on a cold-electron bolometer
}

This document has been downloaded from Chalmers Publication Library (CPL). It is the author's version of a work that was accepted for publication in:

JETP Letters (ISSN: 00213640)

Citation for the published paper:

Tarasov, M. ; Kuzmin, L. (2005) "Concept of a mixer based on a cold-electron bolometer". JETP Letters, vol. 81(10), pp. 538-541.

http://dx.doi.org/10.1134/1.1996765

Downloaded from: http://publications.lib.chalmers.se/publication/106631

Notice: Changes introduced as a result of publishing processes such as copy-editing and formatting may not be reflected in this document. For a definitive version of this work, please refer to the published source. Please note that access to the published version might require a subscription.

Chalmers Publication Library (CPL) offers the possibility of retrieving research publications produced at Chalmers University of Technology. It covers all types of publications: articles, dissertations, licentiate theses, masters theses, conference papers, reports etc. Since 2006 it is the official tool for Chalmers official publication statistics. To ensure that Chalmers research results are disseminated as widely as possible, an Open Access Policy has been adopted.

The CPL service is administrated and maintained by Chalmers Library. 


\title{
Concept of a Mixer Based on a Cold-Electron Bolometer
}

\author{
M. Tarasov ${ }^{1,3}$ and L. Kuz' min $^{2,3}$ \\ ${ }^{1}$ Institute of Radio Engineering and Electronics, Russian Academy of Sciences, Moscow, 125009 Russia \\ e-mail: tarasov@hitech.cplire.ru \\ ${ }^{2}$ Institute of Nuclear Physics, Moscow State University, Moscow, 119992 Russia \\ ${ }^{3}$ Chalmers University of Technology, SE41296 Gothenburg, Sweden \\ Received December 27, 2004; in final form, April 25, 2005
}

\begin{abstract}
A phase-sensitive terahertz heterodyne mixer of a new type based on a cold-electron bolometer is proposed. In this mixer, a normal-metal thin-film absorber is connected to a planar antenna via superconductor-insulatornormal metal (SIN) tunnel junctions, thus forming a SINIS structure. The SINIS mixer combines the advantages of a hot-electron bolometer (HEB), such as a high signal frequency at a small local oscillator power, with the advantages of an SIS mixer, including low noise level, a high intermediate frequency, and wide working temperature range (up to a critical temperature of the superconductor). In contrast to the HEB and SIS mixers, the proposed device is less sensitive to external magnetic noise and exhibits no additional noise related to the superconducting transition and the Josephson effect. ( 2005 Pleiades Publishing, Inc.
\end{abstract}

PACS numbers: 74.50.+r, 85.25.Pb

Introduction. At present, high-sensitivity superconductor mixers for the terahertz frequency range are of two main types, which are based on a superconductor-insulator-superconductor (SIS) structure and a hotelectron bolometer (HEB). The SIS mixer allows a noise temperature lower than $100 \mathrm{~K}$ (which is equivalent to several quantum noise limits $\left.T_{n}^{q}=h f / k\right)$ to be reached at frequencies below $1 \mathrm{THz}$ [1]. However, above $700 \mathrm{GHz}$ (which corresponds to the energy gap for niobium) or twice as high a frequency (for niobium nitride), the noise temperature of SIS mixers increases sharply. In HEB mixers [2], the noise temperatures at frequencies above $1 \mathrm{THz}$ are on the order of $1000 \mathrm{~K}$ and can be approximately evaluated as $T_{n}=10 \mathrm{hf} / \mathrm{k}$ [3]. The principle of the HEB mixer operation is substantially different from that of traditional modulation type mixers (such as the SIS mixer), in which the conductivity of a nonlinear element is modulated at a local oscillator (LO) frequency. The conductivity of an HEB mixer cannot be modulated at such a high LO frequency because this structure is too inertial and cannot follow a terahertz heterodyne frequency. Instead, this slow mixer detects an average interference signal, this resembling the situation in a Fourier spectrometer, where an acoustooptical detector or some other slow detector measures an interference signal at the output of a Michelson interferometer. Both SIS and HEB type mixers have their own advantages and drawbacks with respect to the frequency range, sensitivity to thermal and magnetic fluctuations, excess noise, and losses.

Recently, a cold-electron bolometer (CEB) was proposed [4] and successfully implemented as the incoherent detector with a noise-equivalent power (NEP) of
$10^{-18} \mathrm{~W} / \mathrm{Hz}^{1 / 2}$. This device offers a number of advantages, the most important being the possibility of electron cooling, which provides an increase in the response signal and a decrease in the noise level. This possibility opens the way to the creation of low-noise detectors of both incoherent and phase-sensitive types. Such a CEB mixer design combines the elements of SIS and HEB structures, comprising a thin-film absorber connected to a planar antenna via superconductor-insulator-normal metal (SIN) tunneling junctions. In contrast to an incoherent CEB operating at millikelvin temperatures, CEB mixers operate in the region of conventional helium temperatures. This circumstance increases the response frequency from $10-100 \mathrm{MHz}$ up to $1-10 \mathrm{GHz}$, which corresponds to the standard heterodyne frequencies.

Bolometric mixer operation principle. A power mixer can be considered as the combination of a power detector and an interferometer, in which the interference of the detected and heterodyne signals leads to the appearance of an interference component of the detected power. In the typical case, whereby the LO power is much higher than that of the signal to be detected, we can describe the output signal as

$$
\begin{gathered}
E(t)=\cos \omega t+\alpha \cos (\omega+\delta) t \\
=(1-\alpha) \cos \omega t+\alpha[\cos \omega t+\cos (\omega+\delta) t]=E 1+E 2 .
\end{gathered}
$$

Let us consider the second term in the case when the two amplitudes are equal:

$$
\cos \omega t+\cos (\omega+\delta) t=2 \cos \frac{2 \omega+\delta}{2} t \cos \frac{\delta}{2} t .
$$


This interference signal, representing a combination of two waves, heats the absorber. The total power absorbed in the bolometer at an arbitrary ratio of the component powers is

$$
\begin{gathered}
P=\overline{\frac{E_{1}^{2}}{R}+\frac{E_{2}^{2}}{R}} \\
=\frac{(1-\alpha)^{2}}{R} \cos ^{2} \omega t+\frac{4 \alpha^{2}}{R} \cos ^{2}\left(\frac{2 \omega+\delta}{2} t\right) \cos ^{2} \frac{\delta}{2} t \\
=\frac{(1-\alpha)^{2}}{R}\left(\frac{1-\cos 2 \omega t}{2}\right) \\
+\frac{4 \alpha^{2}}{R}\left(\frac{1-\cos (2 \omega+\delta) t}{2}\right)\left(\frac{1-\cos \delta t}{2}\right) .
\end{gathered}
$$

Averaging at the signal frequency yields

$$
P(t)=\frac{(1-\alpha)^{2}}{2 R}+\frac{\alpha^{2}}{R}-\frac{\alpha^{2}}{R} \cos \delta t .
$$

In the case of equal signal and LO powers, this expression simplifies to

$$
P(t)=\left(\alpha^{2} / R\right)(1-\cos \delta t) .
$$

This formula shows that the absorbed power varies from zero to a certain maximum and the average power is the sum of two equal initial components: $P=\alpha^{2} / 2 R+$ $\alpha^{2} / 2 R=\alpha^{2} / R$.

If the LO power is greater than the signal power, the interference term is automatically formed with only a fraction of the former power corresponding to equal amplitudes. This implies that the LO power can be as small as the signal power. In order to provide for a sufficiently large dynamical range, the LO power should be increased to a level corresponding to the maximum expected signal.

CEB as a mixer. In the case of operation at millikelvin temperatures, the CEB response is rather slow (not exceeding several megahertz), since the electronphonon interaction sharply drops with decreasing temperature. As the phonon temperature grows to a helium level, the interaction increases and its power can be expressed as

$$
P_{\mathrm{ep}}=\Sigma_{V}\left(T_{\mathrm{e}}^{5}-T_{\mathrm{ph}}^{5}\right),
$$

where $\Sigma$ is the material constant, $v$ is the volume, $T_{\mathrm{ph}}$ is the phonon temperature, and $T_{\mathrm{e}}$ is the electron temperature. The effective thermal relaxation time constant in the absence of an electrothermal feedback (electron cooling) can be evaluated using the simple relations $\tau_{0}=C_{\mathrm{v}} / G_{\text {ep }}, \tau=\tau_{0} /(L+1)$, where $C_{\mathrm{v}}=v \gamma T_{\mathrm{e}}$ is the absorber heat capacity, $G_{\mathrm{ep}}=5 \Sigma T_{\mathrm{e}}^{4}$ is the electronphonon thermal conductivity, and $L=G_{\text {cool }} / G_{\text {ep }}$ is the electrothermal feedback gain. Numerical estimates of the thermal time constant yield $10 \mu \mathrm{s}$ at $100 \mathrm{mK}$ and $150 \mathrm{ps}$ at $4.2 \mathrm{~K}$. The electron cooling reduces this value by a factor of 10-100, depending on the cooling efficiency. As a result, the intermediate frequency cutoff may reach $10 \mathrm{GHz}$, which corresponds to the working range of intermediate frequency amplifiers used in coherent receivers for radio astronomy, where the noise temperature does not exceed $10 \mathrm{~K}$.

The power conversion factor can be evaluated proceeding from the basic relations for the electron cooling: if the input heating signal power $P_{\text {sig }}$ is fully compensated by electron cooling, the electron temperature remains constant. Since the energy removed per electron of the cooling current is $k T$, we have $P_{\text {cool }}=P_{\text {sig }}=$ $k T \Delta I / e$ or $I=e P_{\text {sig }} / k T$. The power dissipated in the intermediate frequency load can be estimated as resulting from simple Joule heating by the intermediate-frequency current at a bias voltage close to the gap voltage. For two SIN junctions connected in series, this yields $P_{\mathrm{IF}}=2 V_{\Delta} \Delta I=2 e V_{\Delta} P_{\text {sig }} /(k T)$. Then, the power gain is

$$
G=P_{\mathrm{IF}} / P_{\text {sig }}=2 e V_{\Delta} / k T=e V_{2 \Delta} / k T .
$$

This value differs from a minimum of $3 \mathrm{~dB}$ for the losses of any classical mixer. Then, the possible power gain for a niobium bolometer at helium temperatures can be estimated at $G_{\text {mix }}=9$. Thus, we have a significant gain rather than a loss.

The noise characteristics can be evaluated to the first approximation proceeding from a shot noise of the SIN junction at the mixer output. We take the input noise to be zero (for the input signal, the mixer is simply a matched resistive load equal to the resistance of the metallic thin-film absorber). The current noise at the output, $I_{\mathrm{n}}^{2}=2 e I \Delta f$, can be converted into power as $P_{\mathrm{n}} \approx$ $2 e I R \Delta f \approx 2 e V_{\Delta} \Delta f=k T_{\mathrm{n}} \Delta f$. This yields the output noise temperature $T_{\mathrm{n}}^{\text {out }}=e V_{2 \Delta} / k$, which is about $30 \mathrm{~K}$ for niobium. Taking into account the above power gain of $G=$ $e V_{2 \Delta} / k T$, we obtain a very optimistic estimate for the noise temperature converted to input: $T_{\mathrm{n}}=T$.

Another important advantage of the CEB mixer is that matching of the input signal power is achieved much simpler than that in SIS mixers. Indeed, in our case, there is no need to compensate for the intrinsic capacitance of the tunnel junctions because the impedance due to this capacitance is much lower than the real impedance (resistance) of the metal absorber, which can be readily made equal to the real impedance of the complementary planar antenna on a dielectric substrate (amounting approximately to $70 \Omega$ ). Thus, the exact impedance matching is provided in a broad frequency range. The matching at an intermediate frequency is much like that in SIS structures and has been well developed for SIS mixers.

In order to evaluate the resistances and capacitances of niobium SINIS mixers, we can use the standard char- 


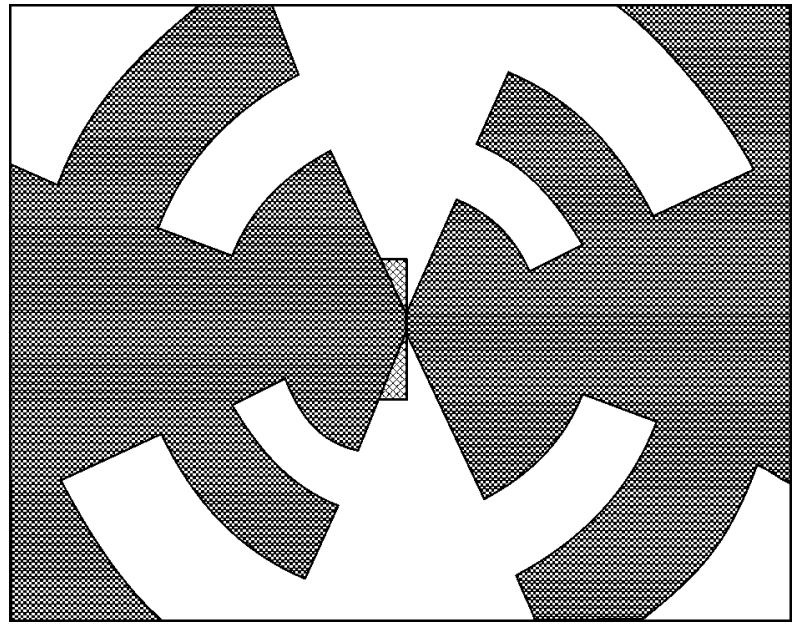

Fig. 1. General view of the SINIS bolometer integrated with a log-periodic planar antenna (bright field corresponds to metallization layers).

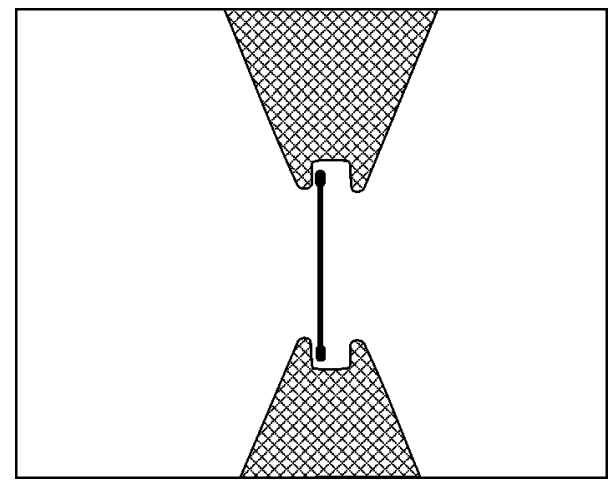

Fig. 2. Central part of a planar antenna with the SINIS bolometer.

acteristics of SIS junctions with aluminum oxide barriers [5]. These structures are characterized by the product $R_{n} A\left(R_{n}\right.$ is the normal resistance and $A$ is the area of the junction) within 25-30 $\Omega \mu \mathrm{m}^{2}$, which corresponds to a current density of $7-8 \mathrm{kA} / \mathrm{cm}^{2}$ and a specific capacitance of $70 \mathrm{fF} / \mu \mathrm{m}^{2}$. Thus, for a junction area of $1 \mu \mathrm{m}^{2}$, the resistance can be about $30 \Omega$ and the capacitance, about $70 \mathrm{fF}$. At an intermediate frequency of $4.5 \mathrm{GHz}$, the capacitive impedance amounts to about $500 \Omega$ and the normal resistance, to $30 \Omega$. The absorber resistance to be matched with a planar antenna at a given signal frequency is about $70 \Omega$. As a result, the matching at the intermediate frequency has to be provided between a $130-\Omega$ impedance of the SINIS structure and a $50-\Omega$ impedance of the standard cooled intermediate frequency amplifier, which is readily achieved using a quarter-wave coplanar matching transformer with a characteristic impedance of $80 \Omega$. By implementing the tunnel junction technology [6] and using an AlN barrier with $R_{n} A=1 \Omega \mu \mathrm{m}^{2}$, one can completely eliminate the problem of matching at the intermediate frequency. Indeed, a contribution due to the serial resistance of the tunnel junction in this case decreases down to several ohms, and the impedance at the intermediate frequency is determined by the absorber resistance that can be taken equal to $70 \Omega$ for matching to the planar antenna at the signal frequency.

CEB development and investigation. In order to study the limiting characteristics of CEBs, we have developed the first generation of such devices using a standard technology for the thermal deposition of tunnel junctions via a suspended two-layer resist mask. An aluminum film was deposited at an angle to the substrate and then oxidized so as to obtain a tunneling barrier. A copper absorber stripe with a length of $10 \mu \mathrm{m}$ was deposited perpendicularly to the substrate. The sample structures were formed on oxidized silicon substrates by means of electron-beam lithography followed by thermal deposition via a mask. In the first step, a 160-nm-thick Cr-Au-Pd layer was deposited in order to obtain contact pads and planar antennas. Then, the bolometer structure proper was formed via a twolayer PMMA copolymer mask in a single technological cycle. A bottom 70-nm-thick superconducting aluminum layer was obtained by thermal deposition at a rate of $0.3 \mathrm{~nm} / \mathrm{s}$. The tunneling barrier was formed by thermal oxidation for $2 \mathrm{~min}$ in oxygen at a pressure of $10 \mathrm{~Pa}$. Finally, a 70-nm-thick copper absorber film was deposited on top of the structure. As a result, the tunnel junctions were formed in the regions of overlap between the oxidized aluminum electrodes and the absorber film. The junction areas were $0.5 \times 0.7$ and $0.2 \times 0.3 \mu \mathrm{m}^{2}$.

The bolometers were integrated with log-periodic and double dipole antennas. The general view of the typical SINIS structure with a log-periodic antenna is presented in Fig. 1. Figure 2 shows a magnified image of the tunneling junction. A limiting NEP value of $10^{-18} \mathrm{~W} / \mathrm{Hz}^{1 / 2}$ was determined by dc measurements in a cryostat at $50 \mathrm{mK}$. The current, voltage, and spectral responses were measured at a temperature of $280 \mathrm{mK}$ using a cryostat with an optical window. As can be seen from the spectrum presented in Fig. 3, the proposed SINIS bolometer is capable of detecting radiation in the submillimeter wavelength range with frequencies up to about $2 \mathrm{THz}$, which is significantly higher than the frequency corresponding to the energy gap for aluminum. The NEP at $280 \mathrm{mK}$ was $2 \times 10^{-17} \mathrm{~W} / \mathrm{Hz}^{1 / 2}$. The sample topology and the procedure of measurements are described in more detail elsewhere [7]. The time constant of aluminum samples measured using the input signal modulation technique was on the order of a microsecond at $280 \mathrm{mK}$. The characteristic time of the electron-phonon relaxation in copper is usually estimated using the relation $\tau_{\mathrm{ep}}=20 / T^{3}$, according to which 


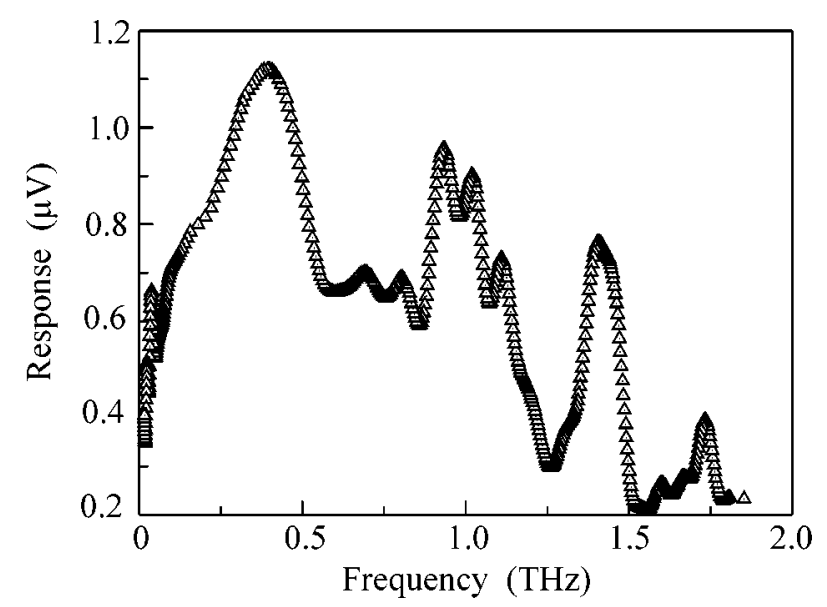

Fig. 3. Spectral response of the bolometer in the terahertz frequency range.

$\tau_{\text {ep }}$ is about $1 \mu$ s at $300 \mathrm{mK}$ and decreases to $0.36 \mathrm{~ns}$ at $4.2 \mathrm{~K}$. In order to increase the intermediate frequency in CEB mixers of the second generation, we intend to switch from $\mathrm{Al}-\mathrm{AlOx}-\mathrm{Cu}$ to $\mathrm{Nb}-\mathrm{AlOx}-\mathrm{Pd}$ structures and measure their characteristics at liquid helium temperature $(4.2 \mathrm{~K})$. These changes will allow us to increase the intermediate frequency above $1 \mathrm{GHz}$. According to our estimates, by selecting the absorber material, changing the film thickness, and introducing electrothermal feedback, it will be possible to further increase the intermediate frequency up to $10 \mathrm{GHz}$.

In conclusion, we have experimentally demonstrated the implementation of a SINIS structure as an incoherent detector. Based on an analysis of the bolometer characteristics, we propose to use CEBs as coherent detectors of radiation in the terahertz frequency range. These bolometers reduce the level of requirements to a cryogenic system and offer advantages over the existing SIS and HEB mixers with respect to the signal frequency, response intensity, and noise level.

This work was supported in part by INTAS (grant no. 01-686), Vetenskapsradet (Swedish Research Council), and Kungl Vetenskapsakademien (Royal Swedish Academy of Sciences).

\section{REFERENCES}

1. A. Karpov, D. Miller, F. Rice, et al., Proc. SPIE 5498, 616 (2004).

2. E. M. Gershenzon, M. E. Gershenzon, G. N. Gol'tsman, et al., Zh. Tekh. Fiz. 59 (2), 111 (1989) [Sov. Phys. Tech. Phys. 34, 195 (1989)].

3. T. M. Klapwijk, R. Barends, J. R. Gao, et al., Proc. SPIE 5498, 129 (2004).

4. L. Kuzmin, I. Devyatov, and D. Golubev, Proc. SPIE 3465, 193 (1998).

5. L. Filippenko, S. Shitov, P. Dmitriev, et al., IEEE Trans. Appl. Supercond. 11, 816 (2001).

6. H. Dmitriev, I. Lapitskaya, L. Filippenko, et al., IEEE Trans. Appl. Supercond. 13, 107 (2003).

7. M. Tarasov, L. Kuz'min, E. Stepantsov, et al., Pis'ma Zh. Éksp. Teor. Fiz. 79, 356 (2004) [JETP Lett. 79, 298 (2004)].

Translated by P. Pozdeev 